\title{
Fluorine F 18 Ara-G
}

National Cancer Institute

\section{Source}

National Cancer Institute. Fluorine F18 Ara-G. NCI Thesaurus. Code C120100.

A radioconjug ate composed of the guanosine analog arabinosyl guanine (Ara-G), a highaffinity substrate for both cytoplasmic deoxycytidine kinase (DCK) and mitochondrial deoxyguanosine kinase (dGK), labeled with fluorine $\mathrm{F} 18$, that can potentially be used as a tracer for imaging of activated T-lymphocytes during positron emission tomography (PET). Upon administration, fluorine $\mathrm{F} 18$ ara- $\mathrm{G}$ is taken up by and accumulates in activated T-lymphocytes with high levels of DCK and dGK. After phosphorylation by DCK and dGK, the $18 \mathrm{~F}$ moiety can be visualized by PET imaging. Fluorine F 18 ara-G can potentially be used as an imaging agent to detect anti-tumor immune responses and to predict the therapeutic efficacy of immunotherapies. DCK and dGK, both nucleoside salvage pathway enzymes, are overexpressed in activated T-cells. 